\title{
Risk and Uncertainty in Anesthesia
}

\author{
Roman Pohorecki $^{{ }^{*}}$, Gary E. Hill ${ }^{2}$ \\ ${ }^{1}$ Department of Anesthesiology, Southwest Medical Center, Liberal, USA; ${ }^{2}$ Department of Anesthesiology and Pain Management, \\ The University of Texas Southwestern Medical Center, Dallas, USA. \\ Email: *roman_plains@yahoo.com
}

Received May $17^{\text {th }}$, 2013; revised June $19^{\text {th }}$, 2013; accepted July $15^{\text {th }}, 2013$

Copyright (C 2013 Roman Pohorecki, Gary E. Hill. This is an open access article distributed under the Creative Commons Attribution License, which permits unrestricted use, distribution, and reproduction in any medium, provided the original work is properly cited.

\begin{abstract}
This article discusses risk and uncertainty, both stochastic and epistemic, as it applies to anesthesia. It shows the difficulty in quantifying risk of individual case and the somewhat arbitrary and even incorrect and naïve assignment of risk in individual patient care management. Effective and honest communication remains at the core of physician-patient relationship in discussing, evaluating and managing the individual case for optimum outcome as well as patients' and their families' satisfaction and understanding.
\end{abstract}

Keywords: Risk; Uncertainty; Communication

\section{Introduction}

Risk is an integral part of life that is brought by natural forces as well as human activity. Though it is reasonable to assume that many people pondered on the nature of the risk, one can, somewhat arbitrarily, trace the beginnings to 16th century Italian mathematician and physician Gorelamo Cardano, who was more interested in risk related to gambling than to outcomes in medicine [1]. The assessment of risk of anesthesia became possible only after the introduction of anesthesia record over 100 years ago, which allowed more substantiated and reproducible comparisons [2]. Once risk is quantified it can and should be used to guide the decision process through the meaningful narrative. It is common to express risk as a probability or probability distribution. That method, even in simple models, forces us to make certain assumptions and often tends to obscure the difference between the uncertainty about the model and the uncertainty about the knowledge.

In this review, we attempt to broadly define the risk in anesthesia and discuss the relation between risk and uncertainty. We also want to bring to light some imperfections of human mind that are relevant in addressing risk. The decision process, studied by cognitive psychologists, has innate flaws that, even with obvious data limit our ability to recognize, to address, and to properly react to issues related to risk.

\footnotetext{
"Corresponding author.
}

\section{Risk Measures Often Used in Medicine}

In medicine, the most common practice is the estimation of probability or, equivalently, the relative frequency of some event. There are several measures that can be further derived and we summarize them below.

Absolute risk reduction is a subtraction. It answers the question: how much the risk increases or decreases as a result of treatment? In more general terms: how much does being a member of the group changes the risk when compared to a different group or population?

Another, often used measure in medicine, is a number needed to treat. It is a reciprocal of absolute risk and as the term implies it measures the number of patients that need to be treated to achieve a target outcome. Obviously, the smaller the number the better.

Example. Lets assume that the rate of myocardial infarction in general male population is 0.0217 and in a male population receiving a small dose of aspirin is 0.0126 . The difference is 0.0091 . The number needed to treat to avoid 1 case of infarction is $1 / 0.0091$ or about 110 patients.

Relative risk is a division or a ratio: how many more times is the outcome or characteristic prevalent in one group compared to another. Since it is often reported without base rates of particular variables, it tends to exaggerate the small differences. It is calculated over time to a defined endpoint.

The term hazard ratio is derived from survival analysis. It is a ratio of two outcomes over a period of time. One 
can think about relative risk as a cumulative form of hazard ratio. Alternatively, the hazard ratio is an instantaneous measure of relative risk, determined before the endpoint of the study.

Using the above example of infarction and aspirin we can determine that relative risk reduction due to aspirin is $0.0091 / 0.0217=0.42 \%$ or $42 \%$.

We included those rudimentary calculations here to show that when dealing with relative measures it helps to refer to absolute values or, to so called, base. Otherwise, the exclusive reliance on relative differences may be misleading. The following 2 examples should make it clear.

When the Department of Justice reports [3] that Hispanic registered voters were at least a $46.5 \%$ more likely to lack the necessary ID, one may arrive at conclusion that requiring the ID amounts to discrimination. Fortunately, the DoJ cites also two additional measures: the percentage of ID lacking voters among Hispanics is $6.3 \%$ and among non-Hispanics is $4.3 \%$. The difference, $2 \%$, indeed amounts to $46.5 \%$ (2/4.3).

When it was reported that daily consumption of processed meat increases a risk of death by $13 \%$ [4] it does not mean that after 10 years of such diet one faces certain death. The analysis expanded by David Spiegelhalter translates the relative risk into absolute one and clearly shows that the person eating processed meat daily may live 1 year less than the person who moderates the diet (79 years vs 80 years) [5].

Once the risk is known, and a very large body of such measures is indeed completed, we face immediate obstacle: how to clearly present it.

\section{The Importance of Communication}

Even when we, physicians, develop a fairly good knowledge and a command of assessing risk, we face another very important threshold: how to communicate it clearly to the patients. The difficulty arises from the fact that they constitute a group with diverse literacy and cognitive abilities, most often tainted by some degree of anxiety dictated by their circumstance. In addition language introduces imprecise terms as "likely" or "probable". Even if such statements are supported by more exacting numerical information, the information may be misinterpreted by a large fraction of patients [6]. Nevertheless good risk communication should be the integral part of our strategy.

There are several methods aimed at helping physicians and patients to discuss the subject of risk. One such method is putting things in perspective. If we know the chances of winning any prize in a lottery like Power Ball (1:32), dying of any cause during next year (1:100), being struck by lightning $(1: 280,000)$, we can communicate the estimated risk of anesthesia in reference to those recognizable events [7].

\section{The Importance of Distribution}

The risk may be viewed as acceptable and unacceptable.

When we try to determine the risk of vomiting after laparoscopic cholecystectomy or of bradycardia during colonoscopy, we have to start form counting such events. We may quickly realize that there are two major factors that one needs to consider: prevalence and severity. Most frequently occurring events cary small consequences. On the other hand infrequent events often have large conesquences. The graph below illustrates that concept: events in the increasing order of severity are paired with their corresponding frequency. It shows the events carrying the largest consequences are infrequent. It also helps to visualize that events carrying relatively small conesquences may be unacceptable if they happen often (Figure 1).

The concept of viewing risk as acceptable and unacceptable may refer to different categories of risk. For example, the failure risk of anesthesia machine [8] and, unrelated, the risk of nausea after cholecystectomy [9]. The consequence in each category will form a range from nearly inconsequential to very severe. For example, the consequences of nausea and vomiting may range from nuisance (frequent) to medical emergency in the form of esophageal rupture (rare) [10]. Another example of how visualizing a distribution can enhance the understanding of risk comes from the relation between the heart rate and myocardial oxygen demand as described by Slogoff [11]. The graph below is an idealized relationship between the heart rate and the volume of ischemic left ventricular wall. As the heart rate increases above certain level, so may the volume of ischemic myocardium. It is possible that two different individuals will experience different magnitude of ischemia at the same heart rate, thus calling for individualized control of the heart rate for each patient [12]. In fact, there will be a whole distribution of the results for each heart rate, as shown on the

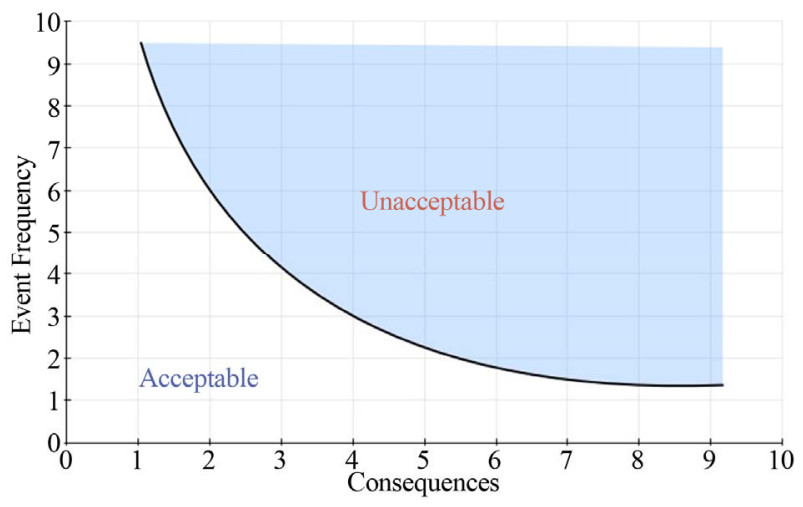

Figure 1. The graph shows the relation between conesquences and the frequency of adverse events. The largest consequences are carried by infrequent events. Units are arbitrary. 
graph below (Figure 2).

The main determinant of the difficulty of risk analysis is the underlying process and its distribution of outcomes. As the complexity of the process increases, its results become more uncertain. Indeed, the term uncertainty as it applies to anesthesia and as it limits our ability to estimate risk will be discussed below.

In terms more general than medicine, one has to realize that the term "risk" encompasses questions related to relatively simple events like the outcome of a die throw to more complicated like risk of arrhythmia as a function of potassium concentration in serum, to very complicated, like Brownian motion. The statistical analysis of a process may reveal that in some cases we may deal with the easy problem that has known probability distribution and applicable tested mathematical methods. On the other side of the spectrum we may encounter limits: the estimation of the distribution parameters are only rough approximations carrying sizable error making risk analysis exceedingly difficult [13]. Because different types of distributions call for slightly different methods to calculate parameters useful in assessing risk, it may be argued that it would be prudent to determine what kind of probability distribution one is dealing with.

Expected, i.e. calculated, occurrence of adverse event requires from us the knowledge (or assumption) of the distribution. We don't want to apply the methodology developed for normal distribution if we are dealing with another type of distribution because it may lead to underestimation of the impact of rare events (contained in the tails of a distribution).

There is, however, a subtle paradox in our ability to determine what exactly is the distribution of outcomes for any process [14]. In order to appropriately determine the type of distribution we need to collect enough data. How many? It depends on the type the distribution that

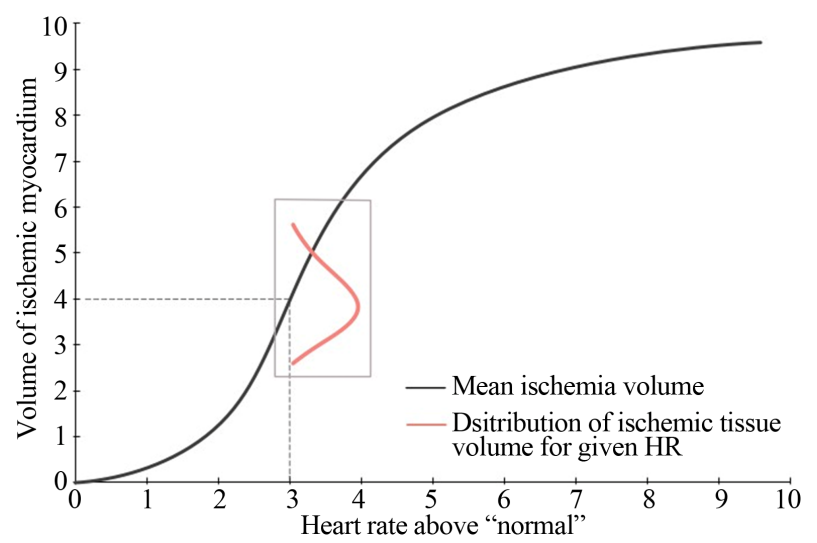

Figure 2. The graph represents idealized relationship between increased heart rate and the mean volume of ischemic myocardium. The units are arbitrary. For every heart rate value above "normal" there is a corresponding distribution of the ischemic tissue volume. will adequately model the studied process, i.e. the very quality that we want to discover. To break out of such circular argument we accept some necessary assumptions supported by the collateral knowledge about the process. The downside of such necessary method is a heightened uncertainty (see below).

It is widely accepted that in biology and medicine the majority of processes under investigation are normally distributed. Most often used standard probability distributions are: for infrequently occurring discrete events it is assumed that the data fit Poisson distribution and for frequently occurring events it is assumed that the data approximate normal distribution. Alternatively, the data may be mathematically transformed, most often in the form of logarithms to "force" fit normal distribution [15]. The importance of the analysis of distribution is illustrated by the study published by Riou and coworkers [16] The authors analyzed cases of a number of blunt trauma victims. They modeled patient's a priori probability distribution of survival showing that it is bimodal. Such finding is important for the assumptions used in subsequent hypothesis testing, like inclusion criteria for further studies. The authors hypothesize that lumping all trauma victims into one study cohort and disregarding the true distribution of survival may have been responsible for the negative results of several earlier studies and trials.

\section{Risk and Uncertainty}

What is simply denoted as risk of anesthesia is in reality a composite of both risk and uncertainty. The meaning of the term "uncertainty" may be a source of confusion. For the purpose of this discussion we will consider two different meanings of this term.

The most common use refers to stochastic uncertainty as in "I am uncertain of a value or a measurement because of a small error". It means that we can not assign an exact value to a parameter in a statistical model that was used for analysis, therefore the parameter is reported with the measure of uncertainty, like confidence limits, standard deviation, etc. In general, the uncertainty here refers to the model and not to reality.

In the second case we restrict the taxonomy to epistemic uncertainty, i.e. the reality, its exact state.

The term refers to an unknown or undetermined part of the risk, to our inability to determine "the state of the world" or in other terms our lack of knowledge. While it is possible to calculate risk, it is impossible to calculate epistemic uncertainty [17]. While risk may be known prior to each event, in the purely uncertain situation the true risks are being discovered as that situation unfolds. The difference between the risk and epistemic uncertainty can be summarized as follows:

1) Risk: The outcome of the process is governed by a known probability distribution and there are known tools 
to analyze it (moments of a distribution: mean, variance, skewness, etc.)

2) Uncertainty: there is not enough knowledge about the process, or the distribution of outcome is unknown, or, in the extreme case, the distribution is probably known but the tools to determine the risk are very limited (as in fat tails distributions).

Both the risk and uncertainty mesh in almost everything we do as physicians.

Uncertainty, as applied to medicine and bracketing both categories, can be appreciated in light of very widespread errors in reported research and a low overall probability of any results being actually correct [18]. Such findings should increase our skepticism, they should serve as a remainder that uncertainty is a big part of what we think we know.

A suitable example of uncertainty includes a burning of the corner of the mouth during tonsillectomy. It may happen when the surgeon uses long non-insulated cautery. Since there is no reliable method to determine the true probability of such event, it is relegated to uncertainty. Similarly, the possibility of explosion in the contemporary anesthesia machine either due to chemical reaction $[19,20]$, or a malfunction of an electronic part [8]. An instructive example of uncertainty in anesthesia is a brief history of perioperative use of beta blocking agents. In the early 1970s the prevailing opinion was that beta blocking agents should be avoided in perioperative period due to their negative inotropism. That point of view changed in the late 1970s and early 1980s when it was observed that sudden cessation of beta blocking agents may lead to increased perioperative incidents of ischemia and heart attacks. It culminated in a series of guidelines formulated by ACC/AHA advocating the use of beta blocking agents [21], and was later incorporated by Center for Medicare Services in its Surgical Care Improvement Project (SCIP). In 2008 the results of a large perioperative ischemic evaluation study (POISE) revealed that indeed the incidence of myocardial infarction is lower in the group treated with metoprolol, but the mortality in treated group was higher: $3.1 \%$ versus $2.3 \%$ $[22,23]$. Metoprolol prevented myocardial infarction in $1.5 \%$ but at the same time it caused excess deaths in $0.8 \%$ and stroke in $0.5 \%$ of the patients. Since POISE studied only acute perioperative treatment with metoprolol, the conclusions do not necessarily apply to chronic treatment.

Similar discussion, currently taking place in anesthesia literature, refers to long term outcomes as a function of cumulative duration of deep hypnotic time as measured by BIS. A number of studies seem to support the association between the cumulative time of deep anesthesia and mortality up to 2 years later. At the same time a number of studies failed to demonstrate that association (for brief reviews see $[24,25]$.

Other examples of considerable uncertainty in anesthesia include regional anesthesia in presence of neurological disease [26], diseases linked to malignant hyperthermia [27], prediction of difficult intubation [28,29], the long term effect of anesthesia on the immune system [30], the platelet count as a restrictive factor for neuroaxial block in obstetrics [31,32], or the hemoglobin levels that would trigger a decision to transfuse [33,34].

Uncertainty about the information or a measure also plays a role in anesthesia practice but under different circumstances. Consider a case of 45 years old male who is being prepared for cholecystectomy. It is reasonable to suspect that he has some degree of coronary atherosclerosis, since the lifetime risk of coronary artery disease events at that age are approximately $40 \%$ [35]. Because the presence of the disease carries a potential impact on the outcome, an anesthesiologist may not be content with the known probability but may wish to increase his confidence of the absence or presence of the disease in this particular case 1 . The frequency of an adverse event may be approximated from a priori large scale epidemiological studies and from personal experience. An anesthesiologist has to determine the probability of an adverse event for a given patient, i.e. assign the numeric value or a linguistic equivalent. It is usually a guess and thus there is a degree of uncertainty about its value. Based on the interview, tests and his own experience the anesthesiologist adjusts the confidence that the probability has a certain value. In other words, as the information is gathered the uncertainty decreases. As the uncertainty decreases, the confidence about a probability of an event changes. The above does not change the true probability of any given event occurring. We often rely on a published mean occurrence or magnitude of an event of interest. We may apply it as a risk measure in a particular situation involving our patient. The fact about such practice worth remembering is that our patient may not be a typical member of the cohort used for the original study. Depending on how atypical he is, the usefulness of the mean value varies. During the evolution of the time series (progression of the case) the initial conclusions are constantly reassessed. The perceived probability may suddenly change during the case. This may occur due to an unknown factor that revealed itself during the case and it constitutes the epistemic uncertainty discussed above. Alternatively, it may be attributed to the process of anesthesia and progression of the surgical procedure.

\section{Compounding Problems}

Multiple co-morbidities and other variables attributed to patient (age, weight, sex, and other genetic factors, etc.) significantly complicate the task to gauge the risk. The assessment of a probability of a single event is an over- 
simplified problem. The reality challenges us to consider more complicated situations in the form of conditional probabilities. Such empirical data are available in the form of different indices.

A conditional probability can be defined as follows: what is the probability of A given the presence (absence) of condition B. There is a lot of epidemiological data accumulated over time based on the above question. Given the dynamic nature of medicine where practice is undergoing a slow but continuous change, and the dynamic nature of societal factors relevant to health, the accumulated data "age" over time and will serve as the estimates only. One may say that the probability estimation is thus conditional on accumulated knowledge at the time of the study. Real life situations pose even more challenging tasks in the form of multiple nested conditional and joint probabilities.

Other factors complicating the assessment of risk include the fact that the more information we seek, the more likely it is that we will include erroneous one. Each test has the inherent limitations summarized as its sensitivity and specificity. As the information is being gathered, its predictive value reaches a plateau. The individual gathering the information will not necessarily gain any new insight or, more importantly, more information will not help him to correct possibly wrong initial conclusion. However, as numerous psychological experiments show, more information improves only self confidence even if the conclusion is incorrect [36,37]. Finally, the process of obtaining additional information intended to reduce risk may have the opposite results: perioperative consultations seem to increase mortality [38].

Variability encountered between individuals administering anesthesia, surgeons, nurses, implemented systems like infection control, medication checks, frequency with which any given case is done in a given hospital, and so on, all add further layers of nested probabilities. All of it impacts the risk.

The psychological constrains on assessing risk and decision making warrant a little more attention. There are several innate mechanisms severely limiting our ability to make rational decisions, to recognize, to address, and to react properly to the issues related to risk. Those limitations have been studied by cognitive psychologists and apply to all of us. Indeed, the recent study estimated the frequency of cognitive errors among anesthesiologists [39] and reported 14 types of cognitive errors such as anchoring ( focusing on one issue at the expense of understanding the whole situation) or premature closure (accepting a diagnosis prematurely). Seven out of 14 errors were made with a frequency higher than $50 \%$. Cognitive and decision errors are made even when the outlining probabilities of some event are known. Kahneman determined the decision weights when people have to make a decision in a situation with an upfront known probability of outcome. There is a strong propensity to overweight small and underweight high probabilities. When the probability of an event is $1 \%$, the corresponding decision weight is 5.5 , when the probability is $5 \%$ the corresponding weight is 13.2 . The opposite is true at the high probability of an event: when it is $80 \%$ the corresponding decision weight is 60.1 , when it is $90 \%$ the weight is only 71.2 , and when it is $99 \%$ the weight is only 91.2. It shows that there is a tendency to deliberate over and emphasize unlikely outcomes but hope for the best when the outcome is most likely unfavorable [40]. Such innate psychological constrains that may or may not be modified by training should be recognized as an additional risk generating factor.

\section{Conclusions}

A very complicated system of interdependencies that we sketched here implicates that risk assessment contains "elements of craft-like judgment". Those craft-like elements are heuristics and professional judgment. In the somewhat narrow meaning, the term professional judgment refers to one's ability to make appropriate decisions under mixed conditions of risk and uncertainty. We acquire that ability by repetitive performance of the same task and by continuous intellectual challenge encountered during postgraduate training. Such repetition allows us to gather the empirical and theoretical evidence about our work, and to develop some ability to implement accumulated experience with a good outcome. It also decreases the variance of outcome, that is, it allows us to develop techniques to assure as uniform outcomes as possible.

The professional judgment embodies the intuitive understanding of possible outcomes without underestimation of the uncertainty. It is in essence our ability to develop a good sense of posterior probabilities related to each individual case. In that sense it is what is defined as subjective probability: our educated guess about how likely is an occurrence of a particular event. There even may be a stark difference between on one hand the ability of an individual to correctly solve an exercise based on Bayes theorem and on the other a correct implementation of that theorem in every day practice. We can rarely or never grasp all the elements relevant to risk estimation in an individual who is about to undergo a particular surgical procedure. Open and honest dialog with our patients based on published literature, accepted practice standards, and our own clinical accumen remains the foundation of our ability to communicate risk to our patients.

\section{REFERENCES}

[1] J. Gullberg, "Probability Theory,” In: J. Gullberg, Ed., 
Mathematics. From the Birth of Numbers, W.W. Norton \& Company, New York, 1997, p. 963.

[2] V. Collins, "Records, Mortality and Medical Legal Considerations,” In: V. Collins, Ed., Principles of Anesthesiology, Lea \& Febiger, Philadelphia, 1976, pp. 31-42.

[3] T. Perez and I. D. Voters, "Legal Opinion,” 2012. www.justice.gov/crt/about/vot/sec_5/ltr/l_031212.php

[4] A. Pan, Q. Sun, A. Bernstein, M. Schulze, J. Manson, M. Stampler, et al., "Red Meat Consumption and Mortality," Archives of Internal Medicine, Vol. 172, No. 7, 2012, pp. 555-563. doi:10.1001/archinternmed.2011.2287

[5] D. Spiegelhalter, "What Does a 13\% Increased Risk of Death Mean?” 2012.

[6] M. Galesic and R. Garcia-Retamero, "Statistical Numeracy for Health. A Cross-Cultural Comparison with Probabilistic National Samples,” JAMA Internal Medicine, Vol. 107, No. 5, 2010, pp. 462-468. doi:10.1001/archinternmed.2009.481

[7] Adams AM, Smith AF. Risk perceptionand communication: Recent developments and implications for anesthesia,. Anaesthesia, Vol. , No. , 2001;56(8):745-55. doi:10.1046/j.1365-2044.2001.02135.x

[8] T. E. Schulte and H. Tinker, "Narkomed 6400 Anesthesia Machine Failure,” Anesthesia \& Analgesia, Vol. 106, No. 3, 2008, pp. 1018-1019. doi:10.1213/ane.0b013e3181632257

[9] J. J. Wang, S. T. Ho, Y. H. Liu, Y. Lee, Y. C. Liao and C. M. Ho, "Dexamethasone Reduces Nausea and Vomiting after Laparoscopic Cholecystectomy,” British Journal of Anaesthesia, Vol. 83, No. 5, 1999, pp. 772-775. doi:10.1093/bja/83.5.772

[10] W. O. Bobo, W. A. Billups and J. Hardy, "Boerhavee’s Syndrome: A Review of sIx Cases of Spontaneous Rupture of the Esophagus Secondary to Vomiting," Annals of Surgery, Vol. 172, No. 6, 1970, pp. 1034-1038. doi:10.1097/00000658-197012000-00017

[11] S. Slogoff and A. Keats, "Does Perioperative Myocardial Ischemia Lead to Postoperative Myocardial Infarction? Anesthesiology, Vol. 62, No. 2, 1985, pp. 107-114. doi:10.1097/00000542-198502000-00002

[12] K. Raby, S. Brull, F. Timimi, S. Akhtar, S. Rosenbaum and C. Naimi and A. Wittermore, "The Effect of Heart Rate Control on Myocardial Ischemia among High-Risk Patients after Vascular Surgery,” Anesthesia \& Analgesia, Vol. 88, No. 3, 1999, pp. 477-482. doi:10.1097/00000539-199903000-00002

[13] R. Weron, "Levy-Stable Distributions Revisited: Tail Index > 2 Does Not Exclude the Levy-Stable Regime," International Journal of Modern Physics, Vol. 12, No. 2, 2001, pp. 209-223. doi:10.1142/S0129183101001614

[14] N. Taleb and A. Pilpel, "On the Unfortunate Problem of Nonobservability of the Probability Distribution,” 2004. www.fooledbyrandomness.com/knowledge.pdf

[15] J. Zar, “Data Transformation,” In: J. Zar, Ed., Biostatistical Analysis, Prentice-Hall, Upper Saddle River, 1984, pp. 236-243.

[16] B. Riou, P. Landais, B. Vivien, P. Stell, I. Labbene and P. Carli, "Distribution of the Probability of Survival Is a
Strategic Issue for Randomized Trials in Critically Ill Patients,” Anesthesiology, Vol. 95, No. 1, 2001, pp. 56-63. doi:10.1097/00000542-200107000-00014

[17] A. Schwartz and G. Bergus, "Embracing Uncertainty,” In: A. Schwartz and G. Bergus, Eds., Medical Decision Making Cambridge University Press, Cambridge, 2008, pp. 55-74. doi:10.1017/CBO9780511722080.007

[18] J. Ioannides, "Why Most Published Research Findings Are False,” PLoS Medicine, Vol. 2, No. 8, 2005, p. E124. doi:10.1371/journal.pmed.0020124

[19] B, Castro, L, Freedman, W, Craig and C. Lynch, "Explosion within an Anesthesia Machine: Baralyme, High Fresh Gas Flow and Sevoflurane Concentration,” Anesthesiology, Vol. 101, No. 2, 2004, pp. 537-539. doi:10.1097/00000542-200408000-00036

[20] J. Wu, J. Previte, E. Adler, T. Myers, J. Ball and J. Gunter, "Spontaneous Ignition, Explosion, and Fire with Sevoflurane and Barium Hydroxide Lime,” Anesthesiology, Vol. 101, No. 2, 2004, pp. 534-537. doi:10.1097/00000542-200408000-00035

[21] K, Eagle, P, Berger, H, Calkins and B. Chaitman, “ACC/ AHA Guidline Update for Perioperative Cardiovascular Evaluation for Noncardiac Surgery-Executive Summary," Circulation, Vol. 105, 2002, pp. 1257-1267.

[22] P. Devereaux, H. Yang, S. Yusuf, G. Guyatt, K. Leslie, J. Villar, et al., "Effects of Extended-Release Metoprolol Succinate in Patients Undergoing Non-Cardiac Surgery (POISE trial): A Randomised Controlled Trial,” Lancet, Vol. 371, No. 9627, 2008, pp. 1839-1847. doi:10.1016/S0140-6736(08)60601-7

[23] J. Sear, J. Giles, G. Howard-Alpe and P. Foex, "Perioperative Beta-Blokade, 2008: What Does POISE Tell Us, and Was Our Earlier Caution Justified?” British Journal of Anaesthesia, Vol. 101, No. 2, 2008, PP. 135-138. doi:10.1093/bja/aen194

[24] T. Monk and B. Weldon, "Anesthetic Depth Is a Predictor of Mortality: It's Time to Take the Next Step," Anesthesiology, Vol. 112, No. 5, 2010, pp. 1070-1072. doi:10.1097/ALN.0b013e3181d5e0eb

[25] C. Kalkman, L. Peelen and K. Moons, "Pick up the Pieces: Depth of Anesthesia and Long-Term Mortality,” Anesthesiology, Vol. 114, No. 3, 2011, pp. 485-487. doi:10.1097/ALN.0b013e31820c2ba2

[26] J. Guay, "First, Do No Harm: Balancing the Risks and Benefits of Regional Anesthesia in Patients with Underlying Neurological Disease," Canadian Journal of Anesthesia, Vol. 55, No. 8, 2008, pp. 489-494. doi:10.1007/BF03016667

[27] R. Litman and H. Rosenberg, "Malignant HypethermiaAssociated Diseases: State of the Art Uncertainty," Anesthesia \& Analgesia, Vol. 109, No. 4, 2009, pp. 1004-1005. doi:10.1213/ane.0b013e3181ae6b96

[28] T. Shiga, Z. Wajima, T. Inoue and A. Sakamoto, "Predicting Difficult Intubation in Apparently Normal Patients,” Anesthesiology, Vol. 103, No. 2, 2005, pp. 429-37. doi:10.1097/00000542-200508000-00027

[29] J. Tse, E. Rimm and A. Hussain, "Predicting Difficult Endotracheal Intubation in Surgical Patients Scheduled for 
General Anesthesia: A Prospective Blind Study,” Anesthesia \& Analgesia, Vol. 81, No. 2, 1995, pp. 254-258. doi:10.1097/00000539-199508000-00008

[30] J. Homburger and S. Meiler, “Anesthesia Drugs, Immunity, and Long-Term Outcome," Current Opinion in Anesthesiology, Vol. 19, No. 4, 2006, pp. 423-428. doi:10.1097/01.aco.0000236143.61593.14

[31] U. M. Stamer, R. W. Stuber and T. M. Wulf, "Contraindications to Regional Anaesthesia in Obstetrics: A Survey of German Practice," International Journal of Obstetrics, Vol. 16, No. 4, 2007, pp. 328-335. doi:10.1016/j.ijoa.2007.05.011

[32] T. Gernsheimer, A. H. James and R. Stasi, "How I Treat Thrombocytopenia in Pregnancy,” Blood, Vol. 121, No. 1, 2013, pp. 38-47. doi:10.1182/blood-2012-08-448944

[33] L. C. Stehling, D. C. Doherty, R. J. Faust, A. G. Greenburg and C. R. Harrisonl, "Practice Guidlines for Blood Component Therapy: A Report by the American Society of Anesthesiologists Task Force on Blood Component Therapy,” Anesthesiology, Vol. 84, No. 3, 1996, pp. 732747. doi:10.1097/00000542-199603000-00032

[34] G. Nutall, B. Brost, R. Connis, J. Gessner, C. Harrison, R. Miller, et al., "Practice Guidelines for Perioperative Blood Transfusion and Adjuvant Therapies: An Updated Report by the American Society of Anesthesiologists Task Force on Perioperative Blood Transfusion and Adjuvant Therapies,” Anesthesiology, Vol. 105, No. 1, 2006, pp. 198-208. doi:10.1097/00000542-200607000-00030
[35] D. Lloyd-Jones, M. Larson, A. Beisera and D. Levy, "Lifetime Risk of Developing Coronary Heart Disease," Lancet, Vol. 353, No. 9147, 1999, pp. 89-92. doi:10.1016/S0140-6736(98)10279-9

[36] S. Oskamp, "Overconfidence in Case-Study Judgments," In: S. Oskamp, Ed., Judgment under Uncertainty: Heuristics and Biases, Cambridge University Press, Cambridge, 2001, pp. 287-293.

[37] E. Berner and M. Graber, "Overconfidence as a Cause of Diagnostic Error in Medicine," American Journal of Medicine, Vol. 121, No. 5, 2008, pp. S2-S23. doi:10.1016/j.amjmed.2008.01.001

[38] D. N. Wijeysundera, P. C. Austin, W. S. Beattie, J. E. Hux and A. Laupacis, "Outcomes and Processes of Care Related to Preoperative Medical Consultation,” Archives Internal Medicine, Vol. 170, No. 15, 2010, pp. 1365-1374. doi:10.1001/archinternmed.2010.204

[39] M. Stiegler, C. Neelankavill, C. Canales and A. Dhilon, "Cognitive Errors Detected in Anesthesiology: A Literature Review and Pilot Study,” British Journal of Anaesthesia, Vol. 108, No. 2, 2012, pp. 229-235. doi:10.1093/bja/aer387

[40] D. Kahneman, “The Fourfold Pattern,” In: D. Kahneman, Ed., Thinking, Fast and Slow, Farrar, Straus and Giroux, New York, 2011, pp. 300-321. 